\title{
RFID Tag Antenna Based Temperature Sensing in the Frequency Domain
}

\author{
R. Bhattacharyya, C. Floerkemeier and S. Sarma \\ Auto ID Labs \\ Massachusetts Institute of Technology \\ Cambridge, MA 02139 \\ Email: rahul_b, floerkem, sesarma@mit.edu
}

\author{
D. Deavours \\ Information and Telecommunications Technologies Center \\ University of Kansas \\ Lawrence, KS 66045 \\ Email: deavours@ittc.ku.edu
}

\begin{abstract}
The efficiency of cold supply chain operations can be improved with pervasive temperature sensing. In this paper, we investigate the design of a low-cost, single-use RFID based temperature threshold sensor that is capable of relating the violation of a temperature threshold to a shift in the optimal operating frequency at which the tag antenna is well matched to the tag IC. This shift is detectable by commercial UHF RFID readers operating in the $902-928 \mathrm{MHz}$ frequency band. We will illustrate how state change information is preserved using a nonelectric memory mechanism that works even in the absence of reader transmitted power. We demonstrate that the sensor works reliably for a read distance of over $3 \mathrm{~m}$ and in noisy environments.
\end{abstract}

\section{INTRODUCTION}

Cold supply chain operations suffer from a lack of visibility with an estimated $30 \%$ perishables being damaged or destroyed somewhere in transit operations [1]. Prior research has shown that cold chain efficiency can be significantly improved by utilizing temperature sensors to monitor ambient temperature conditions [2]. Cold chain operations currently use expensive wireless sensors and for economical reasons, temperature monitoring is done by equipping dedicated places such as cold chain trucks and warehouses and extrapolating this temperature to the goods assumed to be inside. A ideal solution would be to monitor the goods themselves but this requires pervasive temperature sensors that can ideally be inexpensively deployed on every item or pallet passing through the cold chain.

RFID technology represents a pragmatic choice for the design of temperature sensors since it offers many desirable attributes such as standardized communication protocols [3], low cost tags [4], and adoption by major US retail companies as a track and trace solution. This can be highlighted by the emergence and advent of RFID based temperature sensing solutions in the market today. Existing solutions typically rely on RFID tags which feature a battery and a dedicated temperature sensor [5] [6].

More recently, researchers have proposed sensors which use the RFID tag antenna as a temperature sensor [7] to reduce the cost of the sensor device by using conventional UHF RFID tags. Temperature violations are detected as changes in the received signal strength from the RFID tag. Since the received signal strength is not only influenced by the temperature violations but also by reader-tag separation and fading and multi-path issues, these approaches employ a second RFID tag as a reference tag. The second RFID tag increases the size and cost of the sensor.

In this paper, we investigate whether temperature threshold violations can be related to a controlled shift in the optimal operating frequency of a standard UHF RFID tag so that the shift is detectable by commercial UHF RFID readers operating in the American unlicensed ISM band (902-928 $\mathrm{MHz}$ ). Since frequency domain measurements are more noise tolerant and independent of reader-tag separation, operating in the frequency domain would eliminate the need to use a second tag. Our contribution is two-fold. We present a sensor design that converts temperature violations to a change of tag antenna properties using a shape memory polymer and a metal plate. The device is intended to be single-use, which means that the sensor cannot be reset once the temperature threshold is violated. We also present a design and design methodology for an UHF RFID tag antenna that shifts its optimum operating frequency within the $902-928 \mathrm{MHz}$ band when a temperature threshold is exceeded.

Section II highlights some of the previous research and commercial instances of RFID based temperature sensing. Section III then discusses the proposed sensing principle. Section IV describes a sensor prototype that is used to implement the sensing principle, while Section V summarizes a set of performance requirements for our tag antenna and the process used to design such an antenna. Section VI discusses the results of field testing the antenna design and finally Section VII summarizes the key findings of the study and outlines scope for future work.

\section{RELATED WORK}

Temperature indicator labels [8] are commonly used in the perishable goods supply chain today and relate temperature violations to a color change. While these sensors are cheap, monitoring them is labor intensive and requires direct line of sight. Electrical transduction techniques are much more promising from the point of view of automated monitoring and there have been several prior attempts to develop these kinds of temperature sensors. For example, Fletcher et al. propose the design of reversible and single-use temperature threshold 
sensors as well as continuous monitoring temperature sensors by relating temperature to changes in the frequency characteristics of magnetic materials [9]. These sensors are very low cost and operate over a temperature range of $20-80^{\circ} \mathrm{C}$. However, they do not have an inherent unique identifier and have a short read range of a few inches. In related work, the authors also illustrate how electromagnetically responsive material structures can be used to design low cost force and position sensors [10].

Wireless temperature sensing using RFID has also been gaining traction in the recent years. For instance, KSW offers an RFID HF tag sensor capable of logging 720 data points and which has a range of $25 \mathrm{~cm}$ [5]. Similarly, PowerID offers the PowerTMP UHF temperature sensor tag that is capable of storing up to 20 temperature threshold violations. The PowerTMP sensor tag has the advantage of broadband performance in the $860-960 \mathrm{MHz}$ band, a service life of 2 years and a compact form factor [6]. However, the presence of on-board memory and battery components increase the sensor cost, reducing the scale of sensor deployment for both these types of sensor tags.

Intel Research has designed the WISP as a passive UHF front end to low power temperature sensor electronics [11]. The WISP offers a read range of a few meters, works well over a broad temperature range of $70^{\circ} \mathrm{C}$ and is a lower cost alternative to semi-passive RFID sensing. However, events like temperature threshold violations would be missed when the sensor is unpowered.

An alternative approach, involves using the RFID tag antenna as a sensing mechanism by relating the change in a physical parameter to a change in the electrical properties of a tag. For example, Bhattacharyya et al. examine the use of RFID tags as displacement and temperature threshold sensors by mapping displacements and temperature threshold violations to changes in the tag backscatter power strength [12], [13] as well as to the threshold transmitted power required from the reader to just turn on the tag IC [12]. Siden et al. describe the construction of a humidity sensor based on this paradigm [14], while Marrocco et al. discuss how the dielectric permittivity of different media can be monitored using this concept [15]. In [7] and [13], the authors make use of the fact that RFID performance degrades near metals to permanently detune the tag antenna on violation of a critical temperature threshold. This change in antenna characteristics is effected even when the sensor is not powered by a reader antenna, creating a nonelectric memory mechanism. The design proposed in [7] has the added advantage of better range, smaller form factor and performance that is independent of the material of deployment and sensor placement orientation.

These type of sensors use standard low cost RFID ICs with no additional electronic components and this makes them very low cost. Like other sensors that leverage a passive RFID front end however, this sensing paradigm trades off reduced cost for lack of data storage and a reduced communication range. Furthermore, the accuracy of these sensors is coarser than those based on solid state electronics. The RFID tag backscatter signal is also prone to fading and multipath in the environment of deployment, and sensor measurements based on signal amplitude strength are prone to significant noise. Furthermore, tag signal strength amplitude is dependent on the distance between the reader and tag antennas which is why many of the sensor designs [7], [13], [14] make measurements relative to a reference tag. The requirement of having a reference tag on the sensor packaging increases the form factor of these sensors which is undesirable.

Using a frequency domain-based state detection mechanism, however, would have the advantage of being noise tolerant and independent of the reader-sensor separation - provided the sensor is within read range. Thus, the need for a reference tag is eliminated and this would reduce the sensor form factor. In this paper, we propose the design of an RFID tag antenna sensor that makes use of a shift in the optimal operating frequency at which the tag antenna is well matched to the tag IC to detect a temperature threshold violation. Our tag is designed such that the frequency shifts occur in the 902$928 \mathrm{MHz}$ frequency range making the state change detectable by American UHF RFID readers. In the following sections, we will outline our design philosophy and demonstrate how we make use of commercially available off the shelf LLRP compliant RFID reader equipment for sensor state detection.

\section{SEnsing PRinciple}

The Friis Transmission Equation for free space governs radiative coupling between a transmitting and receiving antenna as outlined in [16] and summarized in a modified form in (1)

$$
P_{\text {chip }}=\frac{P_{t} G_{r} A_{e} \tau}{4 \pi d^{2}}
$$

Here $P_{\text {chip }}$ is the power delivered to the IC, $P_{t}$ is the transmitted power, $G_{r}$ is the reader gain respectively, $A_{e}$ is the effective aperture of the tag antenna and $d$ is the readertag separation distance. One of the key parameters influencing tag read range is the power transfer coefficient $\tau$ outlined in [16] and summarized in (2)

$$
\tau=\frac{4 R_{a} R_{c}}{\left|Z_{a}+Z_{c}\right|^{2}}
$$

Here $R_{a}$ and $R_{c}$ are the antenna and chip resistances and $Z_{a}$ and $Z_{c}$ are the antenna and chip impedances respectively. As we can see, $0 \leq \tau \leq 1$. We know that $\tau=1$ when $Z_{a}=Z_{c}^{*}$. For well matched conditions, the tag read range is longer, or alternatively, for a given read range, the reader needs to transmit less power in order to just detect the tag. It is normally desirable for $\tau$ to be close to 1 over the operating band of 860-960 MHz for world-wide RFID operations.

Both $Z_{a}$ and $Z_{c}$ are functions of the operating frequency of the reader antenna. By causing $Z_{a}$ to change with violation of a temperature threshold, we seek to manipulate the frequencies at which $\tau$ is close to 1 . We therefore intend to design a narrow band RFID tag antenna where there is an appreciable shift in the optimal matching frequencies when a critical temperature threshold is violated. Furthermore, we constrain this frequency shift completely in the 902-928 MHz band of American RFID 
operations. Specifically, we design our sensor to have the following states:

- State 1: $\tau$ is designed to be near 1 for $920-925 \mathrm{MHz}$ and sharply decreases for all other frequencies when the temperature is below a given threshold.

- State 2: If the temperature exceeds the threshold, the optimal frequencies over which $\tau$ is near 1 shifts to the 907-912 MHz range and sharply decreases for all other frequencies.

If we design a tag antenna that exhibits these properties then we are presented with a frequency domain based state detection mechanism that can be implemented on commercially available RFID readers in the Americas: For a given readertag separation, we determine the threshold transmitted power at which the reader just starts detecting the tag sensor and observe the frequency channels on which the tag is responding. If the tag is in State 1, we would expect responses on the 920$925 \mathrm{MHz}$ channels. However, if the tag is in State 2, responses would be in the 907-912 $\mathrm{MHz}$ range. A change in channel responses thus enable us to detect which state the sensor is currently in.

In the next section, we discuss a sensor prototype that will be used to induce a controlled change in $Z_{a}$ upon violation of a temperature threshold and outline what State 1 and State 2 are in the context of this prototype design.

\section{Sensor Prototype Design}

Fig. 1(a) and Fig. 1(b) illustrate the design of the temperature threshold sensor prototype. As seen from the figures, the temperature sensor essentially consists of an RFID tag on a plastic jacket separated from a metal plate. The metal plate serves as an isolator, shielding the tag antenna from the material on which the sensor is deployed. This is important since the tag sensor performance should not arbitrarily be influenced by the material on which it is deployed. The height of the isolation plate from the tag can be controlled using a temperature-actuated switch, such as a shape memory polymer (SMP) [17] designed to transition between the rigid and flexible state at about $7^{\circ} \mathrm{C}$. The temperature range at which the polymer actuates can be controlled by changing the chemistry of the polymer as illustrated by [17].

Fig. 2 illustrates the prototype design concept. As seen from Fig. 2(a), the temperature sensor is placed on the product to be monitored in the cold room and the arming pins are pulled free. As seen from Fig. 2(b), if the temperature remains below $7^{\circ} \mathrm{C}$, the polymer remains in the rigid state and the isolation plate is kept at a constant $\mathrm{h}=10 \mathrm{~mm}$ distance from the RFID tag, which corresponds to State 1 mentioned in Section III. If however, the temperature rises above $7^{\circ} \mathrm{C}$, seen in Fig. 2(c), the polymer starts to actuate and this moves the metal plate closer to the tag. In the completely actuated state, seen in Fig. 2(d), the metal plate is about $\mathrm{h}=3 \mathrm{~mm}$ from the tag, which corresponds to State 2 mentioned in Section III. The shape memory polymer is capable of one way actuation which implies that once the polymer switch actuates to the position outlined in Fig. 2(d),

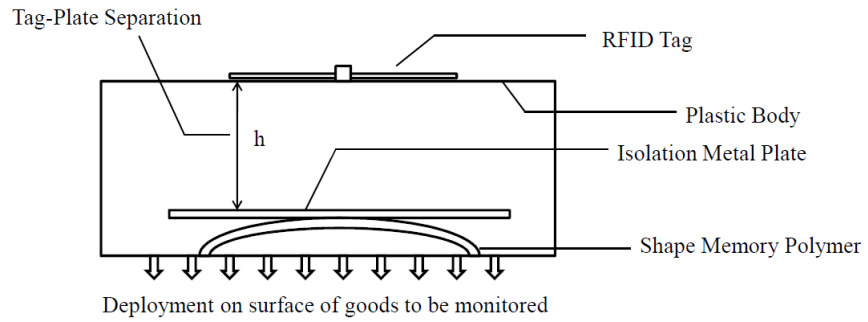

(a) Temperature Sensor in Elevation

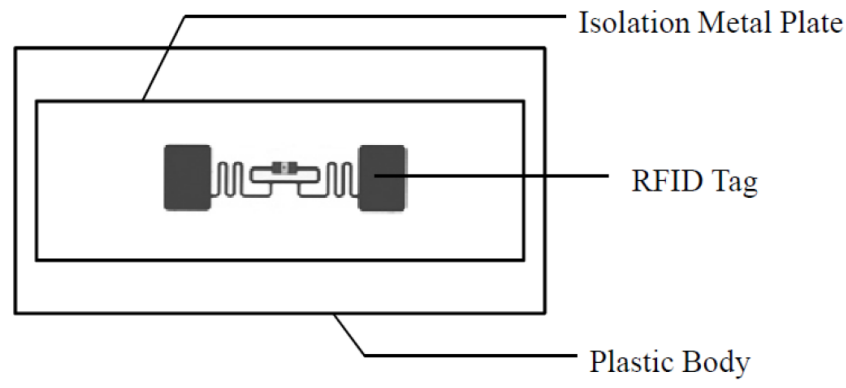

(b) Temperature Sensor in Plan

Fig. 1. Temperature Sensor in (a) Elevation and in (b) Plan

it does not return to the position described in Fig. 2(b) without the application of external stress. This implies that the sensor is designed to be non reusable and disposable. For the purposes of the cold chain, this property is desirable since the purpose of the threshold sensor is to indicate whether a temperature violation occurred at any point in transit in the supply chain. Furthermore, we note that the shape memory polymer triggers a state change even in the absence of reader transmitted power and this creates a non-electric memory for preserving state change information.

There are several other advantages to using an SMP based actuation switch. The SMP will actuate irrespective of the placement orientation and is uninfluenced by gravity allowing the sensor to be placed on the top or sides of the product packaging. In previous work [7], the authors demonstrate sensor performance is agnostic of placement orientation, gravity and material of deployment for a sensor design which is very similar to the one proposed in this paper. Furthermore the quantity of polymer used in the sensor design costs about $\$ 0.1$ which adds a reasonably low cost overhead to the overall sensor design.

The metal plate moving closer to the tag introduces an increased parasitic capacitance to the tag antenna. For a sufficiently narrow-band tag, this would correspond to a reduction in the optimal operating frequency. Precisely controlling the extent of this shift in frequency is the subject of the next section.

\section{Design Methodology}

In this section, we discuss the procedure used to design a tag antenna that exhibits an appropriate shift in optimal operating 

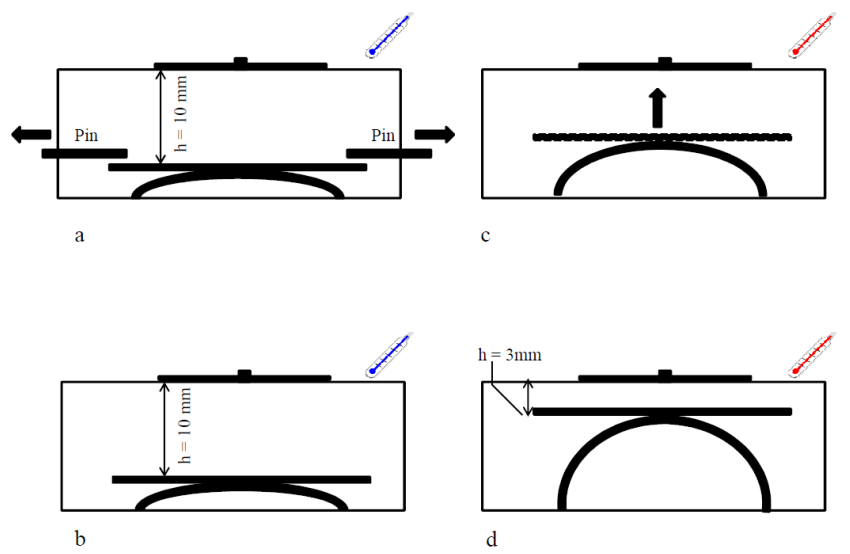

Fig. 2. Temperature Sensor Working

frequency upon violation of the temperature threshold of interest. To aid in the design process, we reiterate the requirements of such an antenna design:

- Narrow Bandwidth: We design the antenna such that the optimal operating frequency band is between 920-925 $\mathrm{MHz}$ in State 1, 907-912 MHz in State 2 and decreases sharply at all other frequencies in the $902-928 \mathrm{MHz}$ range. This is to ensure that we can clearly distinguish between the two states at the threshold transmitted power.

- Frequency Shift in the 902-928 MHz band: The optimal matching frequencies for both states of the sensor, corresponding to the metal plate being 10 and $3 \mathrm{~mm}$ away from the RFID tag antenna respectively, need to be within a $26 \mathrm{MHz}$ band of 902-928 MHz allocated for US RFID operations. This is because commercial RFID readers, which will be utilized to interrogate the tag sensor, are only capable of detecting frequencies in this operating range.

- $\tau$ near 1 at Optimal Matching Frequencies: For a given reader transmitted power, the range over which a tag can be read is directly related to $\tau$ for both States 1 and 2 outlined in Section III. In order for both states of the sensor to be reliably detected over sufficiently long distances, $\tau$ for both states, at the respective optimal frequencies, should be ideally as close to 1 as possible.

\section{A. Selection of an Appropriate Antenna Structure}

We make use of the embedded T-match antenna design since the design has been well characterized and has a limited number of degrees of freedom that we need to manipulate - the length L, width $\mathrm{W}$, slot width s, slot height $\mathrm{w}_{1}$ and slot thickness $t$ as seen in Fig. 3. Deavours et. al present an exhaustive analysis of the T-match antenna explaining how the different geometrical parameters influence antenna impedance [18]. In this section, we briefly highlight only those aspects of their analysis that help us select the geometrical parameters that give us the desired antenna performance.

Our analysis procedure involves finding whether there exists

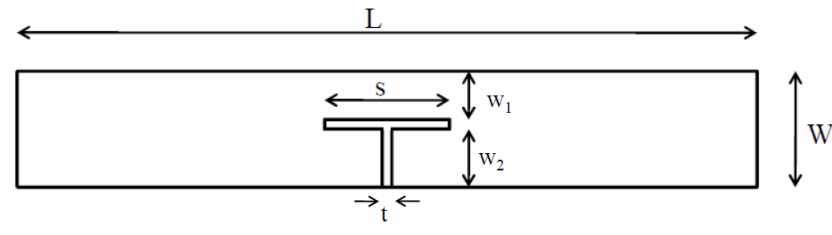

Fig. 3. Embedded T Match Antenna Design

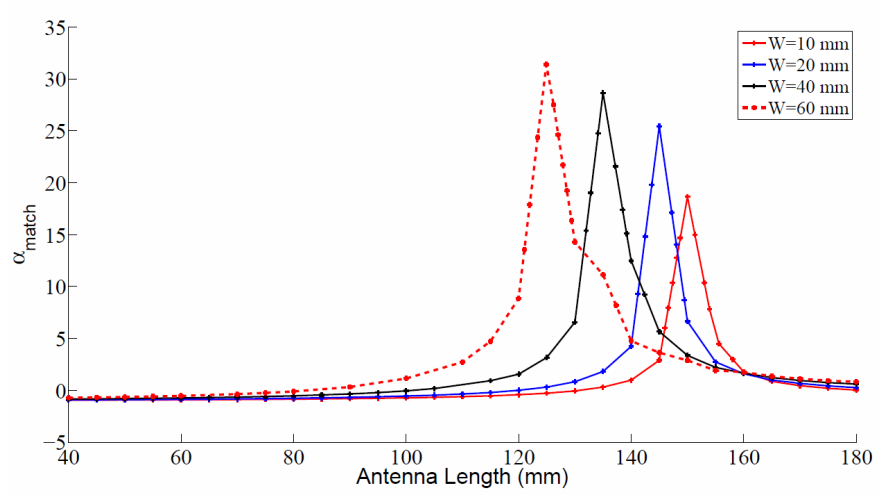

Fig. 4. Variation of $\alpha_{\text {match }}$ with $\mathrm{L}$ for antennas with varying W

a set of parameters $\left(\mathrm{L}, \mathrm{W}, \mathrm{w}_{1}, \mathrm{~s}\right)$ which is well matched to a given IC impedance at $922 \mathrm{MHz}$ when the plate-tag separation is $\mathrm{h}=10 \mathrm{~mm}$ and which is well matched at $907 \mathrm{MHz}$ when $\mathrm{h}=3 \mathrm{~mm}$. Therefore, we first find a set of $\mathrm{L}$ for which impedance matching is feasible at $\mathrm{h}=10 \mathrm{~mm}$.

For a given $\mathrm{L}$ and $\mathrm{W}$ at $922 \mathrm{MHz}$, it is possible to compute the common mode impedance, $Z_{c}=R_{c}+j X_{c}$, of an equivalent dipole antenna at $\mathrm{h}=10 \mathrm{~mm}$ from a metal surface. This can easily be done using numerical simulation software [19]. For a given IC impedance $Z_{I C}=R_{I C}+j X_{I C}$ and $Z_{c}$, Deavours et. al [18] present an expression for values of the splitting factor $\alpha$ that result in an impedance match between the antenna impedance and the IC. We refer to this value of $\alpha$ as $\alpha_{\text {match }}$ as shown in (3)

$$
\alpha_{\text {match }}=\sqrt{\frac{R_{c}\left(R_{I C}^{2}+X_{I C}^{2}\right)}{R_{I C}\left(R_{c}^{2}+X_{c}^{2}\right)}}-1
$$

Fig. 4 illustrates a plot of $\alpha_{\text {match }}$ as a function of $\mathrm{L}$, for different $\mathrm{W}$, when $\mathrm{h}=10 \mathrm{~mm}$ and at $922 \mathrm{MHz}$. As we can see from the figure, for each $\mathrm{W}$, there exists an $\alpha_{\text {match }}$ for a large set of L. To constrain the design space further, we make use of manufacturing tolerances. In their paper, Uda et. al also describe how $\alpha$ varies with antenna geometry [20] as illustrated in (4)

$$
\alpha=\frac{\ln (v)}{\ln (v)-\ln (u)}
$$

where $u=\frac{w_{1}}{w_{2}}$ and $v=\frac{4\left(t+\frac{w_{1}+w_{2}}{2}\right)}{\frac{w_{2}}{2}}$. We can verify that in order to obtain values of $\mathrm{w}_{1}$ and $\mathrm{w}_{2}$ that are larger than 1 $\mathrm{mm}, \frac{1}{3} \leq \alpha \leq 3$. We make use of (4) to impose bounds 


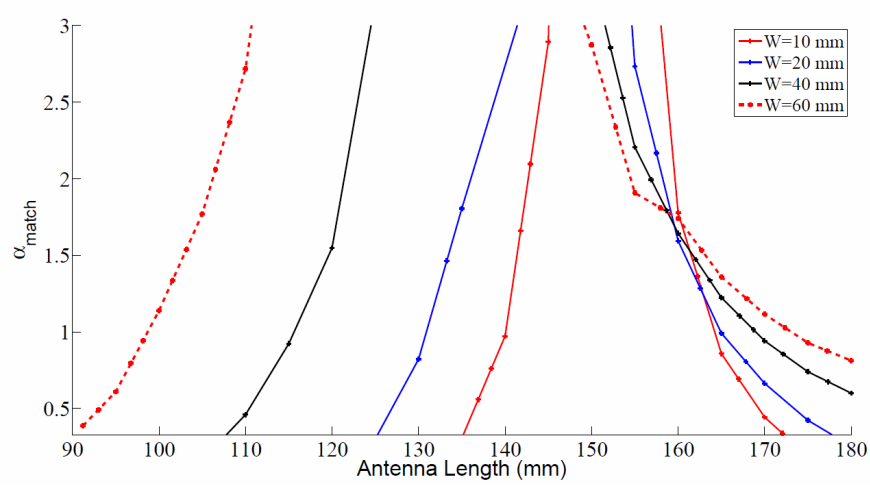

Fig. 5. Variation of $\alpha_{\text {match }}$ with $\mathrm{L}$ considering manufacturing tolerances

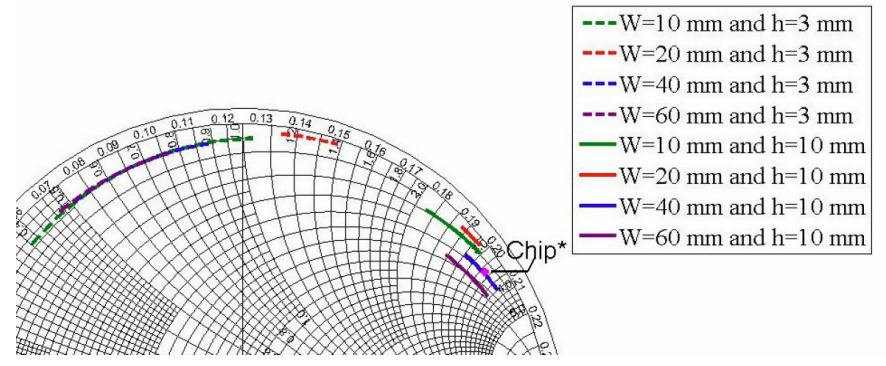

Fig. 6. Antenna Bandwidth Characteristics for Longer Lengths

on the values of $\alpha_{\text {match }}$ to highlight only those values of $\mathrm{L}$ that are feasible from both the manufacturing and impedance matching points of view. This is shown in Fig. 5.

As we can see from the graph, for each $\mathrm{W}$, there exists at least one long and one short $\mathrm{L}$ for which chip matching seems feasible. For example, for the antenna with $\mathrm{W}=40 \mathrm{~mm}$, a short matching length of $110 \mathrm{~mm}$ and a long matching length of 160 $\mathrm{mm}$ is found to be feasible.

\section{B. Antenna Bandwidth Characteristics for Longer Lengths}

As we observe in Fig. 5, for $\mathrm{L}=160 \mathrm{~mm}$, all the antennas have an $\alpha_{\text {match }}$ value of 1.5-1.6. Referring to (4), we determine that $\frac{w 1}{W} \approx 0.7$. Thus for a given width and for a antennametal plane separation of $10 \mathrm{~mm}$, impedance matching to a given tag IC involves varying the slot width, s, shown in Fig. 3. For each antenna width $\mathrm{W}$, we select a slot width that matches the tag antenna to a given tag IC impedance in the 902-928 $\mathrm{MHz}$ frequency range. Once this has been achieved, we change the antenna-metal plane separation to $3 \mathrm{~mm}$, corresponding to State 2 of the sensor, and observe the effect on the antenna impedance. The selection of appropriate $\mathrm{s}$ values is conducted using simulation software [19] and the results are outlined on a Smith Chart shown in Fig. 6.

As we can see from Fig. 6, it is possible to select slots such that for all antenna widths the antenna is reasonably well matched to the complex conjugate of the chip impedance for a tag-metal plate separation of $10 \mathrm{~mm}$. However, when we change the tag-metal plate separation to $3 \mathrm{~mm}$, the tag antennas get severely detuned from the conjugate match position,
TABLE I

TABle of Antenna Dimensions

\begin{tabular}{|l|l|l|l|}
\hline $\mathrm{L}$ & $\mathrm{W}$ & $\mathrm{W} 1$ & $\alpha$ \\
\hline 140 & 10 & 5 & 1.1 \\
\hline 130 & 20 & 7 & 0.8 \\
\hline 112 & 40 & 22 & 1.1 \\
\hline 96 & 60 & 34 & 1.1 \\
\hline
\end{tabular}

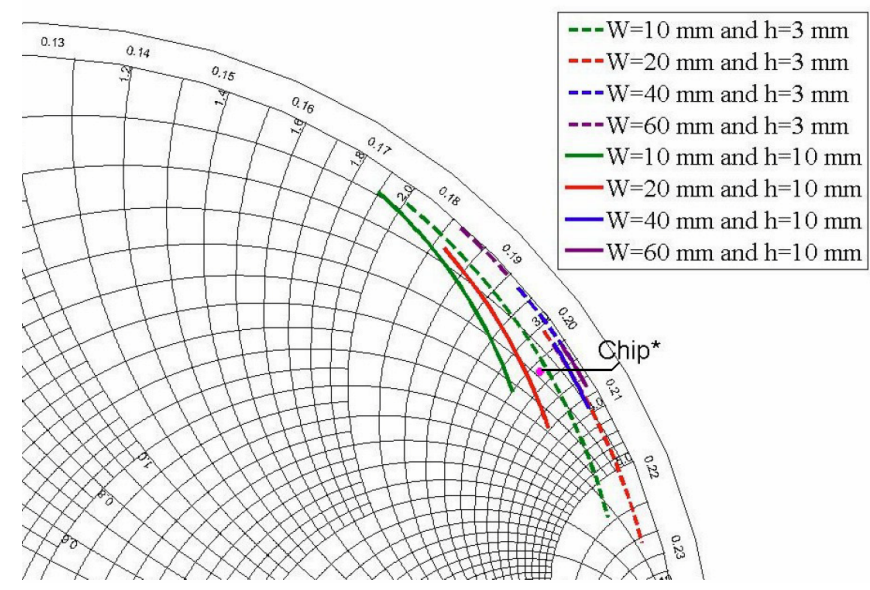

Fig. 7. Antenna Bandwidth Characteristics for Shorter Lengths

drastically reducing $\tau$ in this case. Thus the read range of the tag sensor would be severely limited by virtue of having poor $\tau$ for a tag-metal plate separation of $3 \mathrm{~mm}$. Hence $\mathrm{L}=160 \mathrm{~mm}$ is found not to be a good candidate for our antenna design.

\section{Antenna Bandwidth Characteristics for Shorter Lengths}

Recall from Fig. 5, that there are shorter $\mathrm{L}$ values for which impedance matching is feasible. Table I highlights feasible $\mathrm{L}$ values for antennas of different widths. Repeating the procedure outlined in Section V-B, for a plate-tag separation of $10 \mathrm{~mm}$, we use numerical simulation software to obtain $\mathrm{s}$ values that conjugate match the chip to the antenna in the 902$928 \mathrm{MHz}$ band. Once this is established, we vary the platetag separation to $3 \mathrm{~mm}$ and observe the effect. The results are plotted on a Smith Chart and are shown in Fig. 7.

These results are much more promising. When the tag-metal plate separation changes from $10 \mathrm{~mm}$ to $3 \mathrm{~mm}$, the antenna is detuned very slightly as can be seen by the slight shift in the traces on the Smith Chart when the plate separation is changed. Thus we will be able to observe a slight shift in optimal operating frequency when the sensor changes state. Furthermore, since the detuning is not as severe, we can expect reasonably high $\tau$ values for both sensor states and this improves the read range of the sensor.

In order to select an optimal $\mathrm{W}$ presented in Fig. 7, we plot $\tau$ as a function of frequency for different $\mathrm{W}$ and plate-tag separations. The results are outlined in Fig. 8(a) and Fig. 8(b) respectively. As we can see from the figure, both the antennas corresponding to $\mathrm{W}=10 \mathrm{~mm}$ and $\mathrm{W}=20 \mathrm{~mm}$ exhibit the set of requirements we are looking for - their optimal operating 


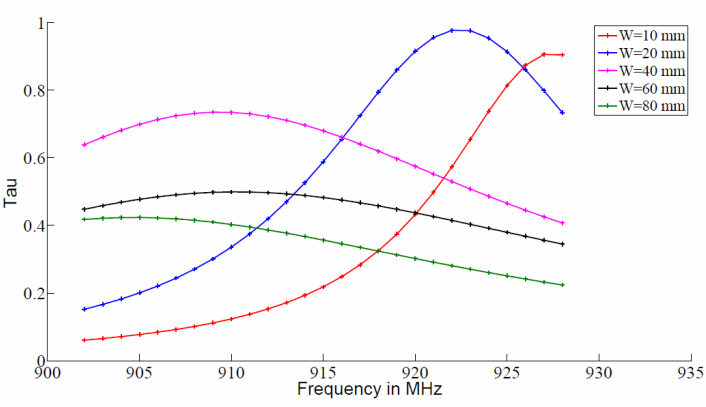

(a) $\tau$ vs Frequency for $\mathrm{h}=10 \mathrm{~mm}$

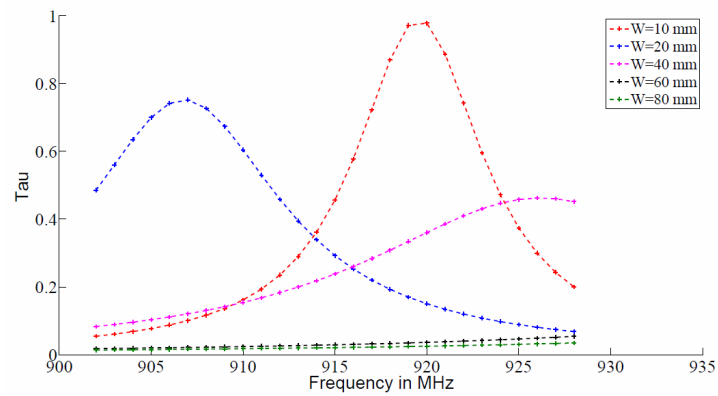

(b) $\tau$ vs Frequency for $\mathrm{h}=3 \mathrm{~mm}$

Fig. 8. $\tau$ vs Frequency for (a) $\mathrm{h}=10 \mathrm{~mm}$ and (b) $\mathrm{h}=3 \mathrm{~mm}$

frequencies shift with a change in position of the metal backplane. The antenna with $\mathrm{W}=10 \mathrm{~mm}$ exhibits a higher power transfer coefficient for the tag-metal plate separation of $3 \mathrm{~mm}$ while the antenna with $\mathrm{W}=20 \mathrm{~mm}$ exhibits a higher power transfer coefficient for the tag-metal plate separation of $10 \mathrm{~mm}$, so we can choose the design appropriately depending upon which state we are more interested in detecting at longer read distances.

We select the tag with $\mathrm{W}=20 \mathrm{~mm}$ since it provides a shift of $15 \mathrm{MHz}$ in peak operating frequency as compared to $8 \mathrm{MHz}$ for the case with $\mathrm{W}=10 \mathrm{~mm}$. The following section outlines the results of field testing this prototype design.

\section{EXPERIMENTAL RESULTS}

Fig. 9 illustrates the antenna prototype that was subjected to field tests. The antenna was manufactured by precision cutting the antenna geometry from a sheet of copper foil. We make use of an Alien Higgs 3 IC [21] and for the purposes of this study assume its nominal impedance to be $Z_{I C}=18-164 j$ at turn on power. We also make an assumption that $Z_{I C}$ remains fairly constant over the $902-928 \mathrm{MHz}$ operating frequency. The IC was bonded to the copper using silver epoxy.

In this section, we discuss the testing procedure employed and examine tag performance in terms of repeatability, read range and operating environment.

\section{A. Testing Procedure}

In this study, we use an Impinj Revolution [22] LLRP compliant RFID reader to detect tag response. By choosing an appropriate implementation of the LLRP protocol stack [23], we can not only extract the operating frequency at the time

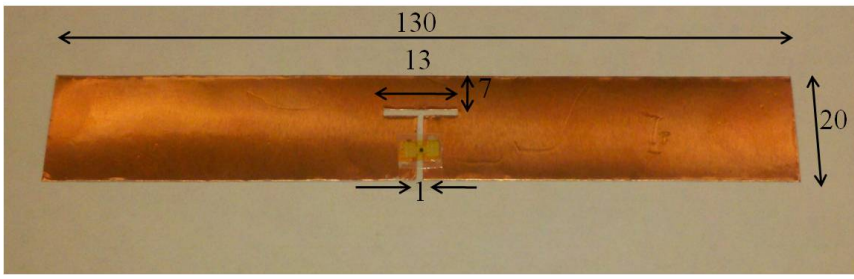

Fig. 9. The Tag Antenna Prototype

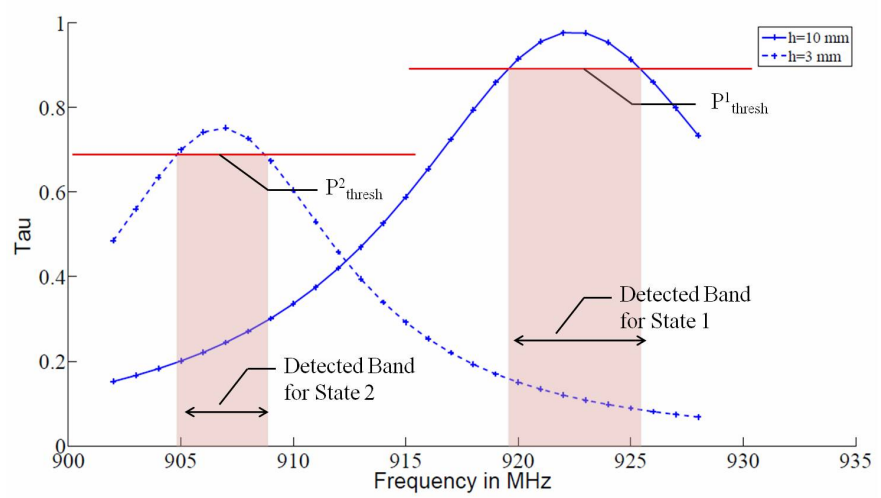

Fig. 10. State Detection Mechanism

of the query round, but also gradually increase the transmitted power $\left(P_{\text {trans }}\right)$ on the reader from $15-32.5 \mathrm{dBm}$ in steps of $0.25 \mathrm{dBm}$. These two features allow us to design a simple testing procedure. We define $P_{\text {thresh }}^{j}(\mathrm{dBm})$ as the threshold power, which is the transmitted power at which the tag is just detected. The index $j$ corresponds to either of the two states 1 or 2 .

To classify state, we proceed by gradually increasing $P_{\text {trans }}$ till we reach $P_{\text {thresh }}^{j}$ and observe the set of channels, $\mathcal{F}_{\text {thresh }}$, on which the tag responds at this power setting. Our classification algorithm is defined as follows:

- Set $j=1$ if $\mathrm{f} \geq 918 \mathrm{MHz} \forall f \in \mathcal{F}_{\text {thresh }}$. In other words, if the sensor is first detected in a small band above 918 $\mathrm{MHz}$ then the tag is in State 1.

- Set $j=2$ if $\mathrm{f} \leq 910 \mathrm{MHz} \forall f \in \mathcal{F}_{\text {thresh }}$. In other words, if the sensor is first detected in a small band below 910 $\mathrm{MHz}$ then the tag is in State 2.

The testing procedure is illustrated in Fig. 10. At a given transmitted power setting, we query the tag antenna for 60 seconds. This is because, RFID readers are mandated to frequency hop randomly ever $400 \mathrm{~ms}$ on $500 \mathrm{kHz}$ channels between $902-928 \mathrm{MHz}$ in the US [24], in order to avoid interference between multiple readers and other RF equipment in the vicinity of the RFID reader. Therefore over a time window of 60 seconds, the reader would have had enough time to query the tag on all 52 channels between $902-928$ MHz. In Section VI-B we present the results of testing the tag antenna's ability to successfully differentiate between the two sensor states using the procedure outlined in this section. 


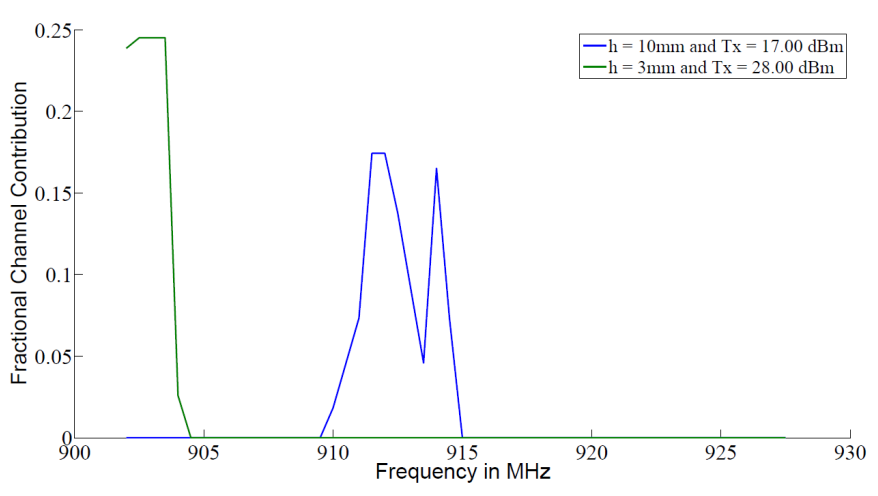

Fig. 11. State Detection using the RFID Reader

\section{B. Efficiency of State Detection}

Fig. 11 illustrates the results of testing for the tag sensor prototype. We conduct the experiment for both states of the sensor and record the frequency contribution, which is the fraction of times the tag was detected in a particular frequency channel for the threshold transmitted power at which the tag was just detected. Our experiment was conducted in an open room, with clear line of sight between the reader and tag antenna. The reader and tag were separated by a distance of $2.5 \mathrm{~m}$. No special anechoic chamber provisions were made to minimize reflections and multipath either. As we can observe from the figure, a clear distinction can be made between the two sensor states at the threshold transmitted power $\left(T_{x}\right)$.

Fig. 12 illustrate the results of repeating the test on 6 replicas of the tag prototype respectively. The purpose of repeating the test multiple times for different replicas is to assess the sensitivity of the antenna performance to variability in the antenna manufacturing process. As we can see, there is some variability between the repetitions although the direction of shift in optimal operating frequency remains consistent. The only anomaly is Replica 6 for which both states register significantly different optimal operating frequencies. We also note that the optimal frequency band is offset by about 5-10 $\mathrm{MHz}$ from the simulation predictions and that the threshold transmitted power varies by as much as $3 \mathrm{dBm}$ between the different replicas. We thus conclude that the tag performance is indeed very sensitive to variability in the manufacturing process and this needs to be taken into account during mass production operations.

\section{Read Range Determination}

It is important to determine the read range over which the tag sensor can be reliably detected for both states of the sensor. For this test, we select one of the replicas (Replica 5) and run the state detection test over different reader-sensor separations. Fig. 13 illustrates the performance of the sensor for a readersensor separation of $2 \mathrm{~m}, 2.5 \mathrm{~m}$ and $3 \mathrm{~m}$.

As we can see from the figure, the sensor can be reliably detected in both states over a distance of $3 \mathrm{~m}$ although there is a variability of 3-5 MHz observed in the position of the frequency bands corresponding to State 1 . The tag IC impedance

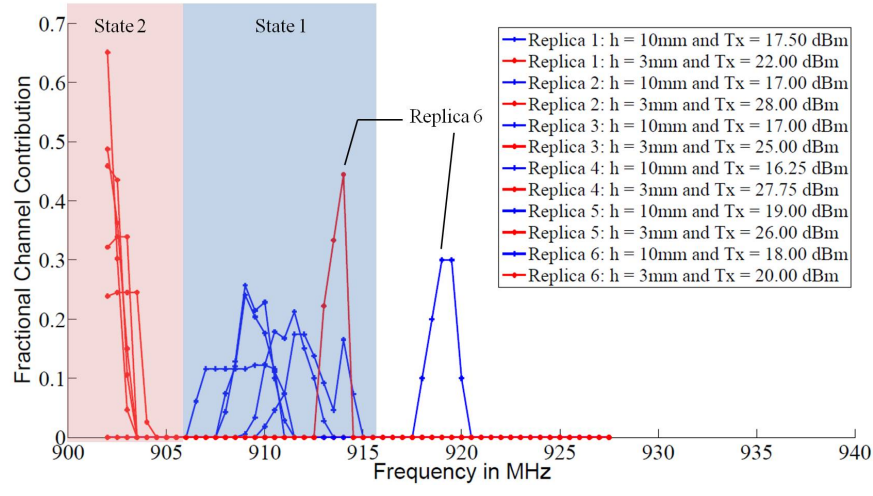

Fig. 12. State Detection on 6 Antenna Replicas

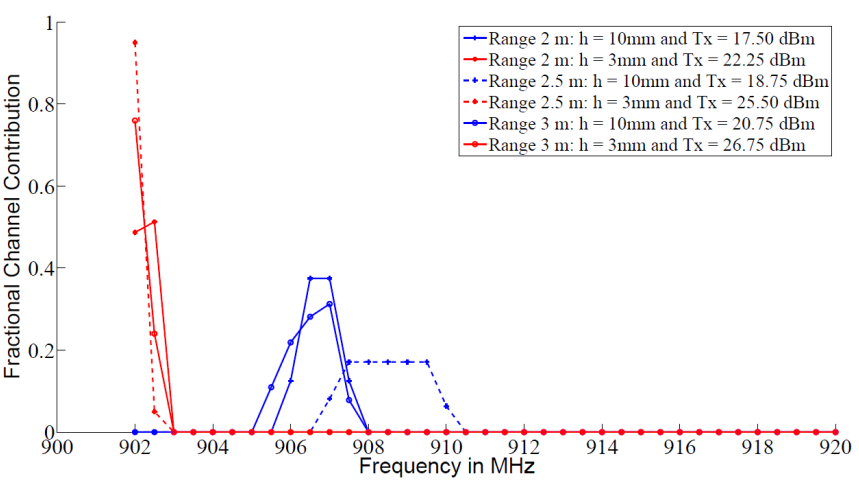

Fig. 13. Sensor Performance over different Read Distances

is a function of transmitted power and it is possible that subtle variations in the threshold transmitted power supplied to the chip brings about this shift. However, these variations are still small compared to the frequency shifts induced by state change and thus state differences can still be successfully interpreted over different ranges.

We also note that the threshold transmitted power for a plate-sensor separation of $3 \mathrm{~mm}$ is $26.75 \mathrm{dBm}$ for a read range of $3 \mathrm{~m}$ which is less than the allowed maximum reader transmitted power of $32.5 \mathrm{dBm}$. This indicates that the maximum read distance of the sensor is larger than $3 \mathrm{~m}$.

\section{Effect of Environment}

The RFID tag sensor is likely to be deployed in several different environments ranging from open warehouse lots to cramped confined storage rooms. It is important to assess how differences in environment affect the tag performance. We examine the tag-sensor performance in a contrived cluttered room with plenty of furniture and metal reflecting surfaces in close proximity to the RFID tag. The only constraint we maintain is a line of sight between the reader and tag antennas. Fig. 14 illustrates the results for a reader-tag separation of $2 \mathrm{~m}$. For the purposes of this experiment we make use of Replica 1.

As we can see from the figure, there is a shift of 3-4 MHz in the frequency band for the sensor in State 1, although it 


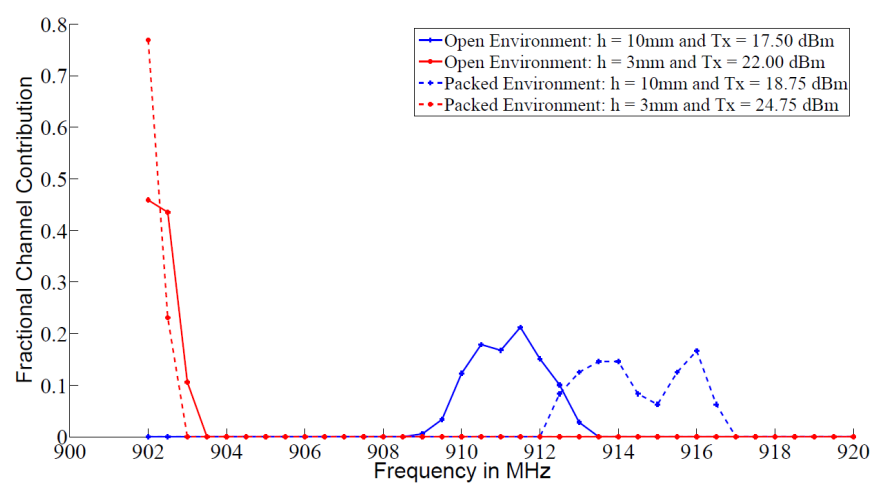

Fig. 14. Sensor Performance in Different Environments

is still possible to differentiate between State 1 and State 2 . Thus the environment does affect tag performance, however by sufficiently separating the frequency bands between State 1 and State 2, we can accommodate the slight variations caused by environmental fluctuations.

\section{CONCLUSIONS}

In this paper, we presented a design methodology for a UHF RFID tag sensor that shifts its optimum operating frequency within the $902-928 \mathrm{MHz}$ band when a temperature threshold is exceeded making binary state detection possible with commercial RFID reader equipment. We illustrated how a temperature actuated shape memory polymer switch can be used as a nonelectric memory mechanism to preserve state changes even in the absence of reader transmitted power. We demonstrated that the frequency domain based state measurements were still discernible in different environments and for read ranges of up to $3 \mathrm{~m}$. This suggests that sensor measurements do not need to be made relative to a reference tag and this will decrease both the cost and the form factor of temperature sensors designed using this principle.

We also discovered that while the antenna can be matched to a given IC at shorter and longer feasible lengths for a T-Match antenna structure, choosing smaller feasible antenna lengths allow us to fulfill our design objectives of having frequency shifts within the 902-928 MHz range while still preserving relatively high $\tau$ values of 0.98 and 0.75 for the two sensor states.

Finally, while we have successfully demonstrated a working sensor design, there are several open issues that we are considering to improve the sensor design and better quantify performance. We have observed that the tag performance is highly susceptible to manufacturing variability. We have also observed that noisy environments and different read distances tend to cause shifts of $3-5 \mathrm{MHz}$ in the optimal operating frequency for State 1 and 2. Quantifying these shifts in a more systematic manner is essential so that we can decide exactly how far apart to space the optimal frequencies for State 1 and 2 , so as to account for these tolerances. Furthermore, while we have investigated sensor performance when the metal plate is at 10 and $3 \mathrm{~mm}$ from the antenna, understanding antenna performance for intermediate separations is important. It is possible that the shape memory polymer might undergo partial actuation during transit and we need to be able to quantify that.

\section{REFERENCES}

[1] A. Dada and F. Thiesse, "Sensor applications in the supply chain: The example of quality-based issuing of perishables," in The Internet of Things, ser. Lecture Notes in Computer Science, C. Floerkemeier, M. Langheinrich, E. Fleisch, F. Mattern, and S. Sarma, Eds. Springer Berlin / Heidelberg, 2008, vol. 4952, pp. 140-154.

[2] A. Ilic, T. Staake, and E. Fleisch, "Using sensor information to reduce the carbon footprint of perishable goods," Pervasive Computing, IEEE, vol. 8, no. 1, pp. $22-29,2009$.

[3] EPC Global Class 1 Generation 2 UHF Air Interface Protocol Standard. http://www.epcglobalinc.org/standards/uhfc1g2.

[4] S. Sarma, "Towards the 5c Tag," Auto ID Labs Whitepaper, 2001.

[5] Variosense - The Smart Card Data Logger. http://www.kswmicrotec.de/index.php?ILNK=Active_RFID_VarioSens.

[6] Affordable Gen 2 Temperature Sensor Tag. http://www.powerid.com/Products/Sensors.aspx.

[7] R. Bhattacharyya, C.V Di Leo, C. Floerkemeier, S. Sarma and L. Anand, "RFID Tag Antenna Based Temperature Sensing using Shape Memory Polymer Actuation," IEEE Sensors Conference Proceedings, pp. 2363 2368, 2010.

[8] DeltaTRAK: Total Cold Chain Management. Temperature Indicator Labels. http://www.deltatrak.com/timetemperatureindicatorlabels.phtml.

[9] R. Fletcher and N. Gershenfeld, "Remotely Interrogated Temperature Sensors Using Magnetic Materials," IEEE Transactions on Magnetics, vol. 36 No. 5 Part 1, pp. 1569-1575, 2000.

[10] R. Fletcher, J. Levitan, J. Rosenberg and N. Gershenfeld, "Applications of Smart Materials to ID Tags and Remote Sensing," Proceedings of Materials Research Society Fall Meeting, vol. 360, 1996.

[11] A. Sample, D. Yeager, P. Powledge, A. Mamishev, and J. Smith, "Design of an rfid-based battery-free programmable sensing platform," Instrumentation and Measurement, IEEE Transactions on, vol. 57, no. 11, pp. $2608-2615,2008$.

[12] R. Bhattacharyya, C. Floerkemeier and S. Sarma, "Towards Tag Antenna Based Sensing - An RFID Displacement Sensor," IEEE International Conference on RFID, pp. 95-102, 2009.

[13] — , "RFID Tag Antenna Based Temperature Sensing," IEEE International Conference on RFID, pp. 8-15, 2010.

[14] J. Siden, X. Zeng, T. Unander, A. Koptyug and H.E Nilsson, "Remote Moisture Sensing utilizing Ordinary RFID Tags," in IEEE Sensors Conference, 2007, pp. $308-311$.

[15] G. Marrocco, L.Mattioni and C. Calabrese, "Multiport Sensor RFIDs for Wireless Passive Sensing of Objects - Basic Theory and Early Results," IEEE Transactions on Antennas and Propagation, vol. 56 No.8, pp. 2691-2702, 2008

[16] K.V.S Rao, P.V. Nikitin and S.F Lam, "Impedance matching concepts in RFID transponder design," in Fourth IEEE Workshop on Automatic Identification Advanced Technologies, 2005, pp. 39 - 42.

[17] D. L. Safranski and K. Gall, "Effect of chemical structure and crosslinking density on the thermo-mechanical properties and toughness of (meth)acrylate shape memory polymer networks," Polymer, vol. 49, no. 20 , pp. $4446-4455,2008$.

[18] N.A Mohammed, K.R Demarest and D.D Deavours, "Analysis and synthesis of UHF RFID antennas using the embedded T-match," in IEEE International Conference on RFID, 2010, pp. 230 -236.

[19] Ansoft: 3D Full Wave Electromagnetic Field Simulation. http://www.ansoft.com/products/hf/hfss/.

[20] S. Uda and Y. Mushaike, "Yagi-Uda Antenna," Sasaki Printing and Publishing Co, pp. 119-131, 1954.

[21] Alien Technologies: The Higgs 3 EPC Class 1 Gen 2 RFID Tag IC. http://www.alientechnology.com/docs/products/DS_H3.pdf.

[22] Impinj Speedway Revolution RFID Reader. http://www.impinj.com/Speedway_Revolution_Reader.aspx.

[23] Fosstrak: Open Source RFID Software Platform. http://fosstrak.org/.

[24] K. Finkenzeller, RFID Handbook: Fundamentals and Applications in Contactless Smart Cards and Identification, 2nd Edition, Ed. New York: John Wiley and Sons Ltd, 2003. 